\title{
sFLT1 in preeclampsia: trophoblast defense against a decidual VEGFA barrage?
}

\author{
S. Lee Adamson \\ Lunenfeld-Tanenbaum Research Institute of Mount Sinai Hospital, Department of Obstetrics and Gynaecology, University of Toronto, Mount Sinai Hospital, Joseph and Wolf Lebovic Health Complex, \\ Toronto, Ontario, Canada.
}

\begin{abstract}
Preeclampsia, a life-threatening complication of human pregnancy, has a spectrum of clinical signs and is likely caused by an array of pathological mechanisms. However, elevated levels of soluble fms-like tyrosine kinase-1 (sFLT1) in the placenta and in the maternal circulation has emerged as a common finding in women with preeclampsia and likely is a causative factor in this disorder. In this issue of the $J C I$, Fan and colleagues provide experimental evidence from both humans and mice that suggests placental trophoblast cells overexpress sFLT1 in self defense against excessive VEGFA produced by maternal decidual cells. The authors' work thus implicates the decidual cells of the mother as the culprit responsible for increased placental expression of sFLT1, a VEGFA antagonist that enters the maternal circulation and consequently induces the clinical signs of preeclampsia.
\end{abstract}

\section{The elusive cause of preeclampsia}

Preeclampsia is a life-threatening complication of human pregnancy and a leading cause of maternal and perinatal morbidity and mortality worldwide (1). The disorder typically arises in the third trimester and is characterized by maternal hypertension and/or signs of organ dysfunction, including proteinuria, thrombocytopenia, impaired liver function, pulmonary edema, and/or cerebral or visual symptoms (1). Although a definitive cause for preeclampsia remains elusive, theories abound (2). It is now considered likely that failure to identify consistent biomarkers (3) or genetic linkages (4) is because preeclampsia is not a single disease (5). Instead, similar clinical signs are elicited by multiple pathological mechanisms. This conclusion is supported by the diversity of clinical signs and placental pathologies among individuals diagnosed with preeclampsia (6), by the diversity in abnormally expressed genes in placentas from patients with preeclampsia (7), and by the wide range of interventions that evoke preeclamptic signs in animal models (8).

Nevertheless, in many preeclamptic pregnancies, maternal circulating levels of soluble fms-like tyrosine kinase-1 (sFLT1) are elevated in late gestation, often before the onset of clinical signs, which suggests that sFLT1 promotes preeclampsia (9). Moreover, in animal models, experimental elevation of sFLT1 can evoke preeclampsia-like signs in late gestation (8). Thus, the discovery that sFLT1 promotes preeclampsia was greeted with considerable excitement (10). Placental overexpression of sFLT1, specifically in the fetal-derived trophoblast cells, was implicated as the underlying cause of preeclampsia (11). The next burning question became, what causes fetal-derived trophoblast cells to overexpress placental sFLT1? In this issue, Fan and colleagues turn the tables and provide a body of work that suggests fetal-derived trophoblast cells overexpress sFLT1 in self defense

Related Article: p. 4941

Conflict of interest: The author has declared that no conflict of interest exists.

Reference information: / Clin Invest. 2014;124(11):4690-4692. doi:10.1172/JCI78532.

against excessive VEGFA produced by maternal decidual cells (12). Thus, the authors' study blames the victim, implicating the decidual cells of the mother as the culprit responsible for disease.

\section{Decidual VEGFA overexpression as a cause of preeclampsia}

Fan et al. examined VEGFA and sFLT1 expression in decidual cells near the placental interface (i.e., the basal plate) in a cohort of women with preeclampsia. The authors found that VEGFA mRNA expression was augmented specifically in maternal decidual cells, whereas sFLT1 mRNA was highly overexpressed in fetal extravillous trophoblast cells that had invaded the decidua - a normal event in placentation (12). These in situ hybridization findings were supported by quantitative RT-PCR, which revealed markedly elevated expression of VEGFA and sFLT1 mRNA in basal plate tissue samples. Fan and colleagues next used adenovirus-based gene delivery and developed a murine model with augmented decidual VEGFA expression. Enhanced decidual VEGFA caused pregnant mice to develop preeclampsiaassociated signs in late gestation, including increased placental sFLT1 protein and mRNA, increased sFLT1 in maternal serum, and maternal hypertension and proteinuria (12). Intriguingly, this etiology appears to define a specific subtype of preeclampsia, given that basal plate biopsies in other cohorts of preeclamptic women have shown no change in VEGFA mRNA (13) or a marked decrease in VEGFA protein and mRNA (14).

In addition to evoking preeclamptic signs in mice, Fan and colleagues found that decidual VEGFA overexpression halved the number of viable fetuses by late gestation, with fetal survivors being markedly growth restricted (12). Fetal losses appeared to occur in midgestation due to hemorrhaging at the maternal-fetal interface, and placental 
histology revealed dilation and engorgement of vessels carrying maternal blood back into the maternal circulation. The authors' work highlights the relevance of their model to preeclampsia; however, they importantly point out that decidual VEGFA overexpression may also underlie other pathological outcomes - including intrauterine growth restriction, early pregnancy loss, and placental abruption - in human pregnancy, where only one conceptus is the norm (12).

To determine whether the ill effects of decidual VEGFA overexpression were caused by the induction of high levels of sFLT1, Fan and colleagues evaluated the effects of lentivirus-mediated knockdown of sFLT1 expression in trophoblast cells. sFLT1 deficiency in trophoblast cells resulted in similar hemorrhaging and venous enlargement, which suggests that increasing the ligand (VEGFA) and reducing the antagonist (sFLT1) promotes similar vascular effects on the venous outflow vessels of the placenta (12). Moreover, the combination of both decidual VEGFA overexpression and sFLT1 knockdown in placental trophoblast caused greater placental pathology and fetal death compared with single manipulation. These results suggest that placental sFLT1 overexpression sequesters VEGFA, reducing placental damage caused by VEGFA overexpression in the adjacent decidua.

Overexpression of sFLT1 only partially rescued phenotypes associated with overexpression of decidual VEGFA, with considerable placental pathology even in venous channels embedded within the sFLT1-overexpressing junctional zone (15, 16). In mice, the junctional zone is considered analogous to the trophoblast cell columns of the basal plate in the human placenta $(17,18)$. Both the human trophoblast columns and the murine junctional zone are at the decidual interface, are devoid of endothelial cells, express high levels of sFLT1, and give rise to invasive cytotrophoblast cells (15, 17-19). Curiously, in contrast to preeclamptic pregnancies, in which maternal plasma sFLT1 may be elevated several-fold (20), the increase in maternal plasma sFLT1 caused by decidual VEGFA overexpression in mice was modest ( 25\%). Furthermore, the increase in sFLT1 occurred after the onset of maternal hypertension and proteinuria.
These observations raise the question of whether maternal preeclamptic signs were actually caused by elevated plasma levels of sFLT1 in this model, or rather by some other mechanism.

\section{Does decidual VEGFA overexpression alter placental endocrine function?}

The decidua and junctional zone are important endocrine organs during rodent pregnancy (21-23). The junctional zone produces multiple prolactins (23), including PL-II (Prl3b1), for example, which in turn influences ovarian endocrine function (24). Thus, in addition to altering placental sFLT1 expression, decidual VEGFA overexpression could alter expression of a myriad of important pregnancy hormones, directly or indirectly. We recently found that reducing VEGFA expression in the junctional zone in all mouse conceptuses in a pregnancy causes maternal hypotension with no change in placental or maternal plasma sFLT1 expression, and there was a surprising increase in maternal plasma VEGFA (25). Given that blood perfusing the junctional zone returns in the maternal venous circulation (16), we speculated that VEGFA deficiency altered endocrine function of the junctional zone, thereby altering endocrine control of the maternal cardiovascular system (25). Similarly, in the model developed by Fan and colleagues (12), it is interesting to question whether decidual VEGFA overexpression altered maternal arterial pressure not by placental sFLT1 release, but by altered decidual and/ or junctional zone endocrine function.

\section{Does impaired spiral artery remodeling influence preeclampsia?}

Although preeclamptic signs were evoked in their mouse model, Fan and colleagues were surprised to find that spiral artery remodeling and trophoblast invasion into the decidua were not impaired (12). This result implies that these abnormalities, which are typically observed in human preeclamptic pregnancies (26), may be independent of increases in placental sFLT1 production and independent of maternal signs of preeclampsia, including maternal hypertension and proteinuria. This observation is consistent with prior work that found blunted spi- ral artery remodeling in diverse human pregnancy pathologies without signs of preeclampsia (26), and other studies that found that failed spiral artery remodeling in mutant mice did not cause preeclamptic signs (27). Thus, the current study adds to accumulating evidence that impaired spiral artery remodeling and reduced trophoblast invasion into the decidua may play associative rather than causative roles in preeclampsia.

\section{Future directions}

In the cohort of human preeclamptic cases studied by Fan and colleagues, decidual VEGFA expression in the basal plate was elevated overall; however, the authors did not report how often a marked increase was observed (12). For example, how many women with preeclampsia had levels $>2$ SD above the mean for women without preeclampsia? Given that prior reports have not observed an elevation in basal plate VEGFA $(13,14)$ and that placental gene expression pathology in preeclampsia has known heterogeneity (7), it is highly likely that an increase in decidual VEGFA explains only a portion of preeclamptic cases. In future studies, it will be valuable to examine a larger cohort of women with preeclampsia in order to determine how common this etiology is and to determine whether there is a correlation between decidual VEGFA and sFLT1 expression, as would be predicted on the basis of the study by Fan et al. (12). It would also be valuable to examine other pathologies associated with human pregnancy in order to assess the specificity of the association between decidual VEGFA and preeclampsia. Moreover, what is the cause for elevated VEGFA in decidual cells in these patients with preeclampsia? Does it predate pregnancy, or is it caused by pathological signals from the implanting conceptus? If the latter is the case, the underlying causative defect in preeclampsia would be put back in the fetal court.

\section{Acknowledgments}

S.L. Adamson acknowledges personnel support as the Anne and Max Tanenbaum Chair in Molecular Medicine at Mount Sinai Hospital as well as operating grant support from the Canadian Institutes for Health Research (grant FRN:93618). 
Address correspondence to: S. Lee Adamson, Lunenfeld-Tanenbaum Research Institute of Mount Sinai Hospital, Department of Obstetrics and Gynaecology, University of Toronto, Mount Sinai Hospital, Joseph and Wolf Lebovic Health Complex, 60 Murray St., Box 42, Toronto, Ontario M5T 3L9, Canada. Phone: 416.586.8377; E-mail:adamson@lunenfeld.ca.

1. Task Force Report on Hypertension in Pregnancy. Washington, DC, USA: The American College of Obstetricians and Gynecologists; 2013.

2. Sibai B, Dekker G, Kupferminc M. Pre-eclampsia. Lancet. 2005;365(9461):785-799.

3. Kuc S, Wortelboer EJ, van Rijn BB, Franx A, Visser GH, Schielen PC. Evaluation of 7 serum biomarkers and uterine artery Doppler ultrasound for first-trimester prediction of preeclampsia: a systematic review. Obstet Gynecol Surv. 2011;66(4):225-239.

4. Jebbink J, Wolters A, Fernando F, Afink G, van der Post J, Ris-Stalpers C. Molecular genetics of preeclampsia and HELLP syndrome - a review. Biochim Biophys Acta. 2012;1822(12):1960-1969.

5. Roberts JM, Bell MJ. If we know so much about preeclampsia, why haven't we cured the disease? J Reprod Immunol. 2013;99(1-2):1-9.

6. Stark MW, Clark L, Craver RD. Histologic differences in placentas of preeclamptic/ eclamptic gestations by birthweight, placental weight, and time of onset. Pediatr Dev Pathol. 2014;17(3):181-189.

7. Cox B, et al. Translational analysis of mouse and human placental protein and mRNA reveals distinct molecular pathologies in human preeclampsia. Mol Cell Proteomics. 2011;10(12):M111.012526.

8. Pennington KA, Schlitt JM, Jackson DL, Schulz
LC, Schust DJ. Preeclampsia: multiple approaches for a multifactorial disease. Dis Model Mech. 2012;5(1):9-18.

9. Kleinrouweler CE, et al. Accuracy of circulating placental growth factor, vascular endothelial growth factor, soluble fms-like tyrosine kinase 1 and soluble endoglin in the prediction of pre-eclampsia: a systematic review and metaanalysis. BJOG. 2012;119(7):778-787.

10. Luttun A, Carmeliet P. Soluble VEGF receptor Flt1: the elusive preeclampsia factor discovered? JClin Invest. 2003;111(5):600-602.

11. Maynard SE, et al. Excess placental soluble fms-like tyrosine kinase 1 (sFlt1) may contribute to endothelial dysfunction, hypertension, and proteinuria in preeclampsia. JClin Invest. 2003;111(5):649-658.

12. Fan $X$, et al. Endometrial VEGF induces placental sFLT1 and leads to pregnancy complications. J Clin Invest. 2014;124(11):4941-4952.

13. Tsatsaris V, et al. Overexpression of the soluble vascular endothelial growth factor receptor in preeclamptic patients: pathophysiological consequences. JClin Endocrinol Metab. 2003;88(11):5555-5563.

14. Kim SC, Park MJ, Joo BS, Joo JK, Suh DS, Lee KS. Decreased expressions of vascular endothelial growth factor and visfatin in the placental bed of pregnancies complicated by preeclampsia. JObstet Gynaecol Res. 2012;38(4):665-673.

15. Hirashima M, Lu Y, Byers L, Rossant J. Trophoblast expression of fms-like tyrosine kinase 1 is not required for the establishment of the maternal-fetal interface in the mouse placenta. Proc Natl Acad Sci U S A. 2003;100(26):15637-15642.

16. Adamson SL, et al. Interactions between trophoblast cells and the maternal and fetal circulation in the mouse placenta. Dev Biol. 2002;250(2):358-373.

17. Georgiades P, Ferguson-Smith AC, Burton GJ. Comparative developmental anatomy of the murine and human definitive placentae. Placenta.
2002;23(1):3-19.

18. Rai A, Cross JC. Development of the hemochorial maternal vascular spaces in the placenta through endothelial and vasculogenic mimicry. Dev Biol. 2014;387(2):131-141.

19. Zhou Y, et al. Vascular endothelial growth factor ligands and receptors that regulate human cytotrophoblast survival are dysregulated in severe preeclampsia and hemolysis, elevated liver enzymes, and low platelets syndrome. Am J Pathol. 2002;160(4):1405-1423.

20. Levine RJ, et al. Circulating angiogenic factors and the risk of preeclampsia. $N$ Engl J Med. 2004;350(7):672-683

21. Knox K, Leuenberger D, Penn AA, Baker JC. Global hormone profiling of murine placenta reveals Secretin expression. Placenta. 2011;32(11):811-816.

22. Shankar K, et al. RNA-seq analysis of the functional compartments within the rat placentation site. Endocrinology. 2012;153(4):1999-2011.

23. Simmons DG, Rawn S, Davies A, Hughes M, Cross JC. Spatial and temporal expression of the 23 murine Prolactin/Placental Lactogen-related genes is not associated with their position in the locus. BMC Genomics. 2008;9:352.

24. Linzer DI, Fisher SJ. The placenta and the prolactin family of hormones: regulation of the physiology of pregnancy. Mol Endocrinol. 1999;13(6):837-840.

25. Li H, et al. Trophoblast-specific reduction of VEGFA alters placental gene expression and maternal cardiovascular function in mice [published online ahead of print August 13, 2014]. Biol Reprod. doi:biolreprod.114.118299.

26. Brosens I, Pijnenborg R, Vercruysse L, Romero R. The "Great Obstetrical Syndromes" are associated with disorders of deep placentation. Am JObstet Gynecol. 2011;204(3):193-201.

27. Burke SD, et al. Spiral arterial remodeling is not essential for normal blood pressure regulation in pregnant mice. Hypertension. 2010;55(3):729-737. 